\title{
Effects of nateglinide and acarbose on glycemic excursions in standardized carbohydrate and mixed-meal tests in drug-naïve type 2 diabetic patients
}

\author{
HAI LI, WENMING XU, JUAN LIU, AILING CHEN, ZHIHONG LIAO and YANBING LI \\ Department of Endocrinology and Diabetes Center, The First Affiliated Hospital of Sun Yat-sen University, \\ Guangzhou, Guangdong 510080, P.R. China
}

Received April 21, 2013; Accepted July 03, 2013

DOI: $10.3892 /$ br.2013.156

\begin{abstract}
The aim of this study was to compare the effects of nateglinide and acarbose on glycemic excursions and postprandial glucose profiles with different types of meals (standardized carbohydrate and mixed meals) in drug-naïve type 2 diabetic patients. A randomized, parallel-group prospective design clinical trial was conducted and a total of 39 drug-naïve patients (16 males and 23 females, aged 56.7 \pm 10.2 years) were enrolled. The patients were randomly divided into group A [nateglinide $120 \mathrm{mg}$ three times daily (t.i.d.), n=19] and group B (acarbose $50 \mathrm{mg}$ t.i.d., $\mathrm{n}=20$ ). The standardized carbohydrate and mixed-meal tests were performed at baseline and at the end of study. Continuous glucose monitoring system (CGMS) data were recorded. Various parameters that measure glucose variability were derived from the CGMS data. In the standardized carbohydrate meal tests, the postprandial glucose excursions (PPGEs) were significantly decreased in the two groups after 12 weeks of treatment $(\mathrm{P}<0.05)$, whereas the decrease was more prominent in the acarbose compared to the nateglinide group $(\mathrm{P}=0.138)$. In the mixed-meal tests, the mean sensor glucose values [24-h mean blood glucose (MBG)] were significantly decreased in the two groups after 12 weeks of treatment $(\mathrm{P}<0.05)$ and the parameters of glucose excursions, including standardized deviation (SD), largest amplitude of glycemic excursion (LAGE), mean of daily differences (MODD) and mean amplitude of glycemic excursion (MAGE), were reduced in the two groups. However, the decreases in SD and LAGE in the nateglinide group were statistically significant, whereas in the acarbose group only the decreases in LAGE were
\end{abstract}

Correspondence to: Professor Yanbing Li, Department of Endocrinology and Diabetes Center, The First Affiliated Hospital of Sun Yat-sen University, 58 Zhong Shan Er Road, Guangzhou, Guangdong 510080, P.R. China

E-mail: easd04lyb@126.com

Key words: type 2 diabetes mellitus, glycose excursion, postprandial glucose, acarbose, nateglinide statistically significant. The efficiency of nateglinide or acarbose in lowering postprandial 120-min hyperglycemia were similar in the standardized carbohydrate meal test. However, acarbose was more efficient in lowering postprandial 30- and 60 -min hyperglycemia $(\mathrm{P}<0.05)$ compared to nateglinide. The fasting and postprandial total cholesterol (TC), triglyceride (TG) and low-density lipoprotein cholesterol (LDL-C) had a tendency to decrease from the baseline after 12 weeks of treatment with nateglinide, whereas fasting and postprandial high-density lipoprotein cholesterol (HDL-C) had a tendency to increase. Acarbose did not affect the fasting or postprandial lipid profiles after 12 weeks of treatment $(\mathrm{P}>0.05)$. In conclusion, nateglinide and acarbose effectively improved postprandial glycemic control, although acarbose was shown to be more efficient in controlling early (30 and $60 \mathrm{~min}$ ) postprandial glucose excursions in the carbohydrate meal test, whereas nateglinide was shown to be superior to acarbose in controlling postprandial glucose excursions in the mixed-meal test.

\section{Introduction}

It has been demonstrated that glucose variability, independent of the glycosylated hemoglobin (HbA1c) levels, may significantly affect the risk for chronic diabetic complications $(1,2)$. Therefore, it is recommended that glucose variability is considered along with HbAlc levels in the management of diabetes $(3,4)$.

It was hypothesized that the postprandial plasma glucose variability is associated with the hypoglycemic drug mechanisms and the types of meals, particularly regarding the proportion of carbohydrate. Therefore, two different anti-diabetic drugs with different mechanisms of action in different types of meal tests were designed. Nateglinide and acarbose specifically target postprandial plasma glucose with different hypoglycemic mechanisms. To the best of our knowledge, there has been no head-to-head trial comparing the effects of these two agents on glycemic excursions in different types of meal tests. Therefore, the different effects of the two drugs on metabolic control and glucose variability were assessed in drug-naïve type 2 diabetic patients, with the aim to determine a treatment strategy that may be more effective in Chinese type 2 diabetic patients. 
Table I. Patient characteristics at baseline.

\begin{tabular}{lccc}
\hline Treatment group & Nateglinide $(\mathrm{n}=19)$ & Acarbose $(\mathrm{n}=20)$ & P-value \\
\hline Age (years) & $54.6 \pm 8.6$ & $58.6 \pm 11.1$ & 0.75 \\
Gender (male/female) & $6 / 13$ & $10 / 10$ & 0.27 \\
BMI & $25.9 \pm 2.6$ & $26.7 \pm 2.9$ & 0.61 \\
SBP $(\mathrm{mmHg})$ & $126.2 \pm 15.1$ & $134.9 \pm 13.4$ & 0.29 \\
DBP $(\mathrm{mmHg})$ & $77.6 \pm 10.0$ & $7.9 \pm 10.2$ & 0.56 \\
HbAlc $(\%)$ & $7.3 \pm 0.6$ & $6.5 \pm 0.8$ & 0.49 \\
FPG $(\mathrm{mmol} / \mathrm{l})$ & $6.5 \pm 1.0$ & $12.4 \pm 1.9$ \\
2-h PPG (mmol/l) & $12.8 \pm 2.3$ & $5.55 \pm 0.76$ \\
TC (mmol/l) & $5.91 \pm 1.40$ & $1.51 \pm 0.99$ & 0.87 \\
TG $(\mathrm{mmol} / \mathrm{l})$ & $1.79 \pm 1.44$ & $3.39 \pm 0.73$ \\
LDL-C (mmol/l) & $3.77 \pm 1.43$ & $1.22 \pm 0.21$ \\
HDL-C (mmol/l) & $1.20 \pm 0.17$ & 0.22 \\
\hline
\end{tabular}

Data are presented as means \pm standardized deviation. BMI, body mass index; SBP, systolic blood pressure; DBP, diastolic blood pressure; HbA1c, glycosylated hemoglobin; FPG, fasting plasma glucose; 2-h PPG, 2-h postprandial plasma glucose; TC, total cholesterol; TG, triglyceride; LDL-C, low-density lipoprotein cholesterol; HDL-C, high-density lipoprotein cholesterol.

\section{Materials and methods}

Study design. A total of 39 drug-naïve type 2 diabetic patients (16 males and 23 females, aged 56.7 \pm 10.2 years) were screened and included in this study. The main inclusion criteria were as follows: fasting plasma glucose $\leq 9 \mathrm{mmol} / \mathrm{l}$, HbA1c $6.5-9.0 \%$ and treatment with diet alone for a minimum of 2 weeks without any concurrent medication prior to enrolment. After 2 weeks of treatment with diet alone, the subjects were randomized into groups A and B. The patients in group A were administered nateglinide $120 \mathrm{mg}$, three times daily (t.i.d.); the patients in group B were administered acarbose $50 \mathrm{mg}$, t.i.d (Fig. 1). A 70-g carbohydrate standardized meal and a consecutive three-day mixed-meal (30 kcal/kg ideal weight/day, based on dietitian recommendations, including $55 \%$ of calories from carbohydrates, $25 \%$ from fat and $20 \%$ from proteins, with a meal distribution of $1 / 5,2 / 5$ and $2 / 5$ ) tests were performed at baseline and at the end of study. In the carbohydrate standardized meal test, blood samples were collected at fasting, 15, $30,60,90$ and $120 \mathrm{~min}$ to calculate the postprandial glucose excursion (PPGE), which was defined as the mean difference between preprandial and postprandial glucose values within $2 \mathrm{~h}$ after the standardized meal. In the consecutive three-day mixed-meal test, the patients underwent continuous glucose monitoring system (CGMS) measurements. They were instructed to perform four glucose calibration measurements and mark the time of each meal by pressing the input button on the CGMS device.

The study protocol was approved by the Ethics Committee of The First Affiliated Hospital of Sun Yat-Sen University and all the subjects provided written informed consent.

Parameters derived from CGMS. All the parameters of continuous glucose monitoring were calculated from each CGMS output, which was extracted using the CGMS 3.0 software package [Medtronic MiniMed, MMT-7310 version 3.0C (3.0.128)]. The mean glucose level was calculated as the mean

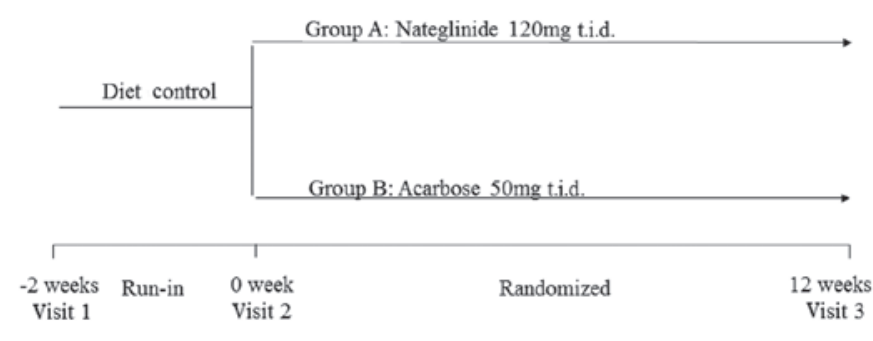

Figure 1. Study design; t.i.d., three times daily.

of all the consecutive sensor readings, from which the standardized deviation (SD) was also calculated. The largest amplitude of glycemic excursion (LAGE) was defined as the maximal sensor glucose level minus the minimal sensor glucose level during each day. The mean amplitude of glycemic excursion (MAGE) was used for assessing the intraday glucose variability. MAGE was calculated by measuring the arithmetic mean of the differences in consecutive peaks and nadirs, which were taken into consideration only if they exceeded one standard of deviation from the mean. The mean of the daily differences (MODD), calculated as the average absolute difference of paired sensor glucose values during two successive 24-h periods, was used to assess the interday glucose variability.

Statistical analyses. Values are expressed as means \pm standard deviation. The statistical significance of variation between means was assessed using the two-tailed paired Student's $\mathrm{t}$-test. $\mathrm{P}<0.05$ was considered to indicate a statistically significant difference.

\section{Results}

Patients. A total of 16 male and 23 female patients with newly diagnosed type 2 diabetes [mean age 56.7 years (SD, 10.2), 
Table II. Parameters of continuous glucose monitoring system in both group at baseline and at 12 weeks.

\begin{tabular}{lllll}
\hline & \multicolumn{2}{c}{ Nateglinide $(\mathrm{n}=19)$} & \multicolumn{2}{c}{ Acarbose $(\mathrm{n}=20)$} \\
\cline { 2 - 4 } Parameters & Baseline & 12 weeks & Baseline & 12 weeks \\
\hline 24-h MBG (mmol/l) & $7.22 \pm 1.14$ & $6.47 \pm 1.17^{\mathrm{a}}$ & $7.27 \pm 1.41$ & $6.65 \pm 1.19^{\mathrm{a}}$ \\
SDBG (mmol/l) & $1.70 \pm 0.82$ & $0.98 \pm 0.48^{\mathrm{a}}$ & $1.85 \pm 0.78$ & $1.28 \pm 0.22$ \\
LAGE (mmol/l) & $7.30 \pm 2.70$ & $4.62 \pm 2.42^{\mathrm{a}}$ & $7.51 \pm 3.5$ & $3.22 \pm 0.79$ \\
MAGE (mmol/l) & $4.58 \pm 2.09$ & $2.34 \pm 1.67^{\mathrm{a}}$ & $4.64 \pm 1.59$ & $1.07 \pm 0.21$ \\
MODD (mmol/l) & $1.31 \pm 0.37$ & $1.01 \pm 0.40$ & $1.47 \pm 0.64$ & \\
\hline
\end{tabular}

Data are presented as mean \pm standardized deviation. 24-h MBG, 24-h mean blood glucose of sensor; SDBD, standardized deviation of all the sensor blood glucose values; MODD, mean of daily differences; LAGE, largest amplitude of glycemic excursion; MAGE, mean amplitude of glycemic excursion. ${ }^{\mathrm{a}} \mathrm{P}<0.05$ compared to baseline in each group.

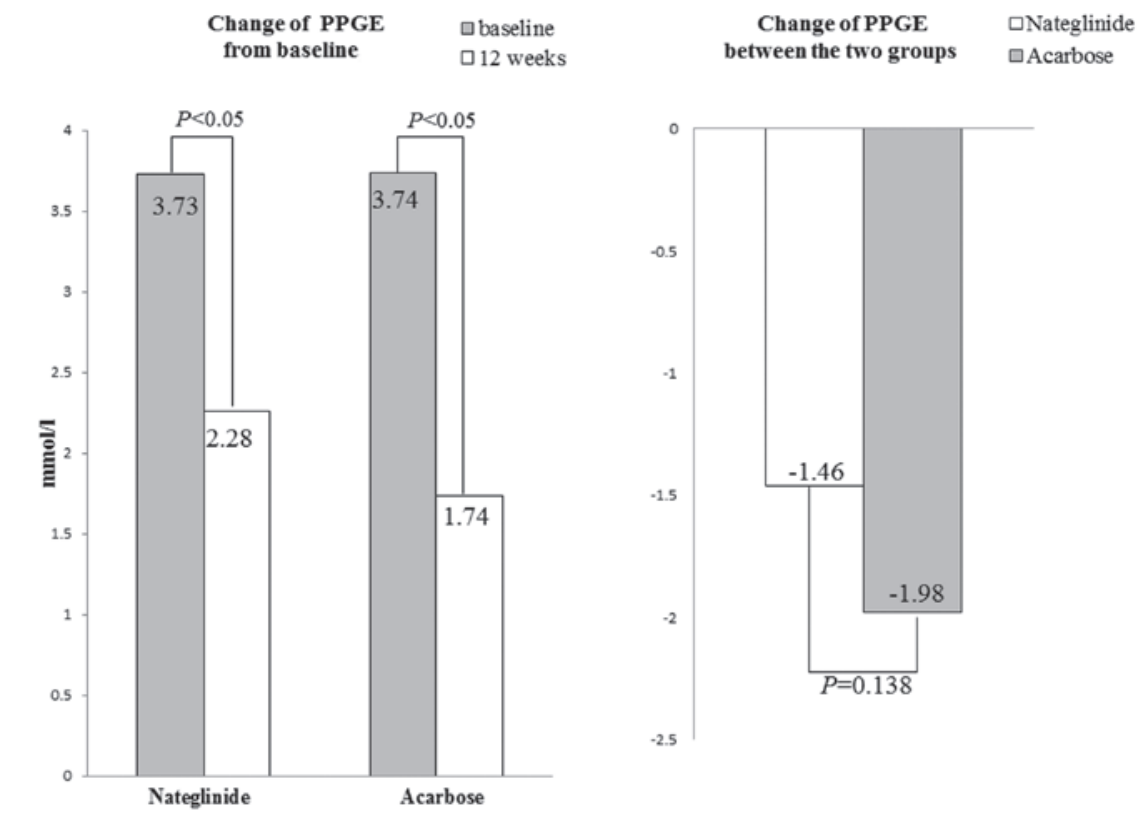

Figure 2. Change of postprandial glucose excursion (PPGE) in the standardized carbohydrate meal test.

body mass index $\left.26.3 \mathrm{~kg} / \mathrm{m}^{2}(\mathrm{SD}, 2.8)\right]$ were randomly assigned to the nateglinide $(n=19)$ and the acarbose groups $(n=20)$. The clinical characteristics, glucose levels and lipid profiles at baseline were similar between the two groups (Table I).

Effects of nateglinide vs. acarbose on postprandial glycemic excursion in the standardized carbohydrate and mixed-meal tests. Standardized carbohydrate meal test: at baseline, the PPGE levels in the nateglinide and acarbose groups were 3.73 and $3.74 \mathrm{mmol} / \mathrm{l}$, respectively, without a statistically significant difference $(\mathrm{P}=0.86)$. After 12 weeks of treatment, PPGE was significantly reduced in the two groups $(\mathrm{P}<0.05)$, with acarbose exerting a more potent effect compared to nateglinide $(-1.98 \pm 1.18$ versus $-1.46 \pm 0.98 \mathrm{mmol} / \mathrm{l}, \mathrm{P}=0.138$ ) (Fig. 2).

Mixed-meal test: the parameters of glucose variability derived from the CGMS were well-matched between the two treatment groups prior to randomization. Analysis of the data calculated from the CGMS output demonstrated that the mean sensor glucose values (24-h MBG) had decreased significantly in the two groups after 12 weeks of treatment. The parameters of glucose excursions, including SD, LAGE, MAGE and MODD, were reduced in the two groups and the reductions in SD, LAGE and MAGE in the nateglinide group were statistically significant. However, in the acarbose group, only the decrease in LAGE was considered to be statistically significant (Table II and Fig. 3).

Effects of nateglinide versus acarbose on postprandial glycemic profiles in the standardized carbohydrate meal test. The efficiencies of nateglinide and acarbose in lowering postprandial 120-min hyperglycemia were similar (-2.35 vs. $-2.52 \mathrm{mmol} / \mathrm{l}$, $\mathrm{P}=0.82$ ). The mean variations in glucose at fasting, 15, 30, 60, 90 and 120 min after a standardized meal from baseline to week 12 in the nateglinide treatment group were -0.32 , $-0.18,-1.02,-2.50,-2.94$ and $-2.35 \mathrm{mmol} / \mathrm{l}$, respectively, with significant differences at 90 and $120 \mathrm{~min}(\mathrm{P}<0.05$, Fig. 4). The corresponding changes in the acarbose treatment group were $-0.47,-1.01,-2.10,-3.2,-3.45$ and $-2.52 \mathrm{mmol} / 1$, respectively, 
Table III. Fasting and postprandial lipid profiles at baseline and at 12 weeks.

\begin{tabular}{|c|c|c|c|c|}
\hline \multirow[b]{2}{*}{ Lipid profiles } & \multicolumn{2}{|c|}{ Nateglinide } & \multicolumn{2}{|c|}{ Acarbose } \\
\hline & Baseline & 12 weeks & Baseline & 12 weeks \\
\hline \multicolumn{5}{|l|}{$\mathrm{TC}(\mathrm{mmol} / \mathrm{l})$} \\
\hline Fasting & $5.91 \pm 1.40$ & $5.46 \pm 1.30^{\mathrm{a}}$ & $5.15 \pm 0.76$ & $5.18 \pm 0.90$ \\
\hline Postprandial & $5.56 \pm 1.398$ & $5.19 \pm 1.18$ & $4.93 \pm 0.70$ & $4.83 \pm 0.84$ \\
\hline \multicolumn{5}{|l|}{$\mathrm{TG}(\mathrm{mmol} / \mathrm{l})$} \\
\hline Fasting & $1.79 \pm 1.44$ & $1.37 \pm 0.63$ & $1.51 \pm 0.99$ & $1.57 \pm 0.83$ \\
\hline Postprandial & $2.13 \pm 1.47$ & $1.61 \pm 0.71$ & $1.75 \pm 1.21$ & $1.79 \pm 1.37$ \\
\hline \multicolumn{5}{|c|}{ LDL-C (mmol/l) } \\
\hline Fasting & $3.77 \pm 1.43$ & $3.73 \pm 1.14$ & $3.39 \pm 0.73$ & $3.37 \pm 0.88$ \\
\hline Postprandial & $3.58 \pm 1.32$ & $3.13 \pm 1.11$ & $3.08 \pm 0.70$ & $3.15 \pm 0.73$ \\
\hline \multicolumn{5}{|c|}{ HDL-C (mmol/l) } \\
\hline Fasting & $1.20 \pm 0.17$ & $1.25 \pm 0.20$ & $1.12 \pm 0.21$ & $1.11 \pm 0.20$ \\
\hline Postprandial & $1.13 \pm 0.16$ & $1.25 \pm 0.28$ & $1.03 \pm 0.19$ & $1.01 \pm 0.16$ \\
\hline
\end{tabular}

Data are presented as means \pm standardized deviation. ${ }^{\mathrm{a}} \mathrm{P}<0.05$ compared to baseline in each group. TC, total cholesterol; TG, triglyceride; LDL-C, low-density lipoprotein cholesterol; HDL-C, high-density lipoprotein cholesterol.
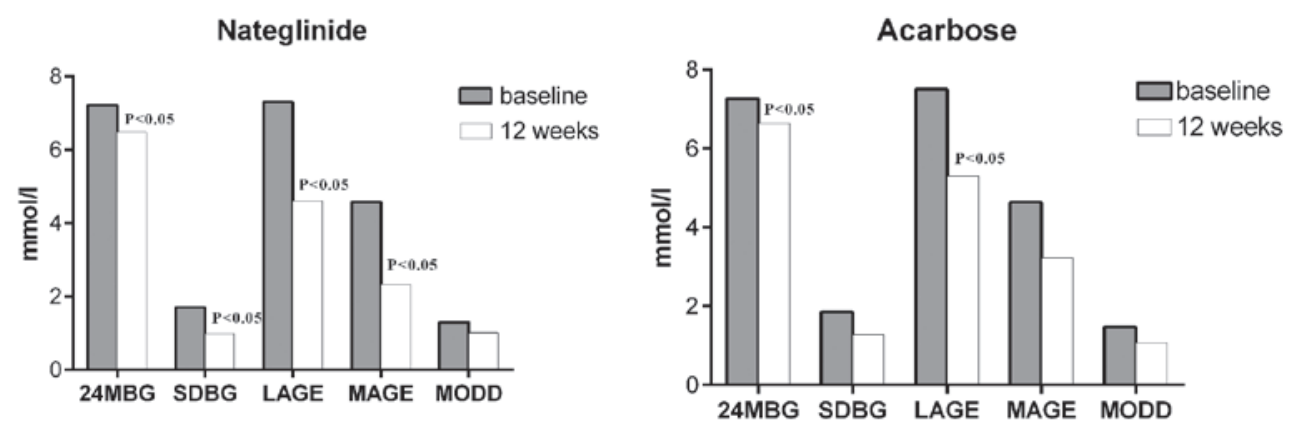

Figure 3. Changes of parameters of postprandial glucose excursion in the mixed-meal test in each group. 24MBG, 24-h mean blood glucose; SDBG, standardized deviation of blood glucose values; LAGE, largest amplitude of glycemic excursion; MAGE, mean amplitude of glycemic excursion; MODD, mean of daily differences.
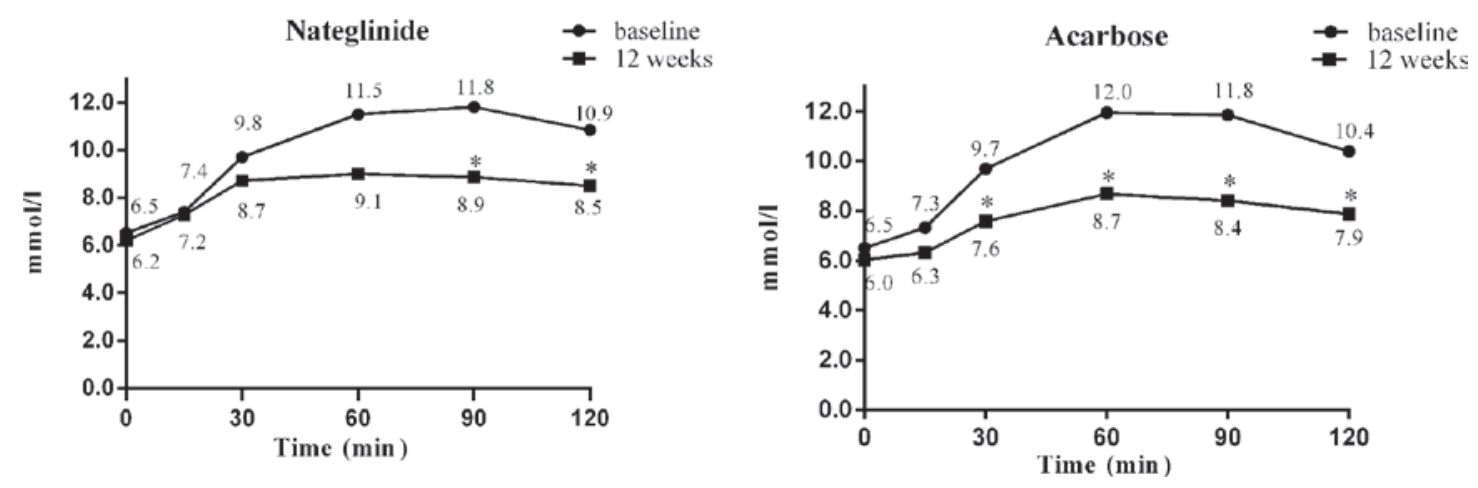

Figure 4. Postprandial glycemic profiles in the standardized carbohydrate meal tests in each group. ${ }^{*} \mathrm{P}<0.05$ compared to baseline in each group.

with significant differences at 30, 60, 90 and 120 min after a standardized meal ( $\mathrm{P}<0.001$, Fig. 4). The intergroup differences in the intragroup changes were significant at 15, 30 and 60 min after a standardized meal, with acarbose treatment being superior to nateglinide treatment at 15,30 and $60 \mathrm{~min}$ after a standardized meal $(\mathrm{P}<0.05)$.
Effects of nateglinide versus acarbose on fasting and postprandial lipid profiles after 12 weeks of treatment. Nateglinide exhibited a tendency to increase the high-density lipoprotein cholesterol (HDL-C) levels and decrease the total cholesterol (TC), low-density lipoprotein cholesterol (LDL-C) and triglyceride (TG) levels after 12 weeks of treatment, with a 
significant reduction $(0.44 \mathrm{mmol} / \mathrm{l})$ of fasting TC compared to baseline $(\mathrm{P}<0.03)$. However, this tendency was not observed in the acarbose group (Table III).

\section{Discussion}

The present study has demonstrated that nateglinide and acarbose exert similar hypoglycemic effects on postprandial 120 min glucose, which was consistent with the findings of previous studies (5-9). We demonstrated that acarbose may be more efficient in controlling early (30 and $60 \mathrm{~min}$ ) postprandial glucose and PPGE in the carbohydrate meal. However, as observed in the mixed-meal test by CGMS, the efficiency of nateglinide in reducing glycemic excursions in mixed meals was superior to that of acarbose.

In type 2 diabetic patients, the early phase of insulin secretion in response to a meal is delayed and blunted, which is crucial in the regulation of postprandial blood glucose levels, with this deficiency resulting in excessive meal-related glucose excursions (10-13). Restoration of early insulin secretion following a meal may exert a beneficial effect on postprandial glucose control and glycemic excursions (14-16). The results of the present study are consistent with those of previous studies, which demonstrated that nateglinide effectively decreases mealtime plasma glucose excursions (17-20). Nateglinide reduces postprandial plasma glucose levels by promoting early short-term insulin secretion, which may exert a beneficial effect on controlling postprandial glucose and excursions.

In the mixed-meal tests evaluated by CGMS, the efficiency of nateglinide in reducing glycemic excursions after a mixed meal was superior to that of acarbose. However, in the standardized carbohydrate meal test acarbose appeared to be superior to nateglinide in controlling PPGE. This paradox may be explained by the differences in the hypoglycemic mechanisms of the two drugs. Acarbose inhibits the breakdown of carbohydrate by binding competitively to $\alpha$-glucosidase; therefore, since in the standardized carbohydrate meal test the patients only ingested carbohydrates, acarbose was more efficient in controlling early $(15,30$ and $60 \mathrm{~min})$ postprandial glucose and PPGE.

The effects of antidiabetic drugs on blood lipid levels were also investigated in this study. It was demonstrated that nateglinide exhibited a tendency to increase HDL-C and decrease LDL-C levels after 12 weeks of treatment. However, acarbose did not affect the fasting or postprandial lipid profiles. It is well known that blood lipid metabolism is affected by various factors and primarily associated with insulin secretion dysfunction in type 2 diabetes mellitus. The effect of nateglinide on lipid profiles may be attributed to its mechanism of action, significantly improving insulin secretion in the early phase. However, acarbose does not exert any direct effect on insulin secretion (21).

In conclusion, the results of our study demonstrated that nateglinide and acarbose may effectively improve postprandial glycemic control. Acarbose may be more effective in controlling early (30 and $60 \mathrm{~min}$ ) postprandial glucose and excursions in the carbohydrate meal test, whereas in the mixed-meal test nateglinide was superior to acarbose in controlling postprandial glucose excursions. In addition, nateglinide exerted beneficial metabolic effects by improving lipid profiles, which may be associated with the restoration of early-phase insulin secretion.

\section{References}

1. Brownlee M and Hirsch IB: Glycemic variability: a hemoglobin A1c-independent risk factor for diabetic complications. JAMA 295: 1707-1708, 2006.

2. Hirsch IB: Glycemic variability: it's not just about A1C anymore! Diabetes Technol Ther 7: 780-783, 2005.

3. Ceriello A and Ihnat MA: 'Glycaemic variability': a new therapeutic challenge in diabetes and the critical care setting. Diabet Med 27: 862-867, 2010

4. Monnier L, Colette C and Owens DR: Glycemic variability: the third component of the dysglycemia in diabetes. Is it important? How to measure it? J Diabetes Sci Technol 2: 1094-1100, 2008.

5. Gribble FM, Manley SE and Levy JC: Randomized dose ranging study of the reduction of fasting and postprandial glucose in type 2 diabetes by nateglinide (A-4166). Diabetes Care 24: $1221-1225,2001$

6. Kim MK, Suk JH, Kwon MJ, et al: Nateglinide and acarbose for postprandial glycemic control after optimizing fasting glucose with insulin glargine in patients with type 2 diabetes. Diabetes Res Clin Pract 92: 322-328, 2011.

7. Hazama Y, Matsuhisa M, Ohtoshi K, et al: Beneficial effects of nateglinide on insulin resistance in type 2 diabetes. Diabetes Res Clin Pract 71: 251-255, 2006.

8. Chiasson JL, Josse RG, Hunt JA, et al: The efficacy of acarbose in the treatment of patients with non-insulin-dependent diabetes mellitus. A multicenter controlled clinical trial. Ann Intern Med 121: 928-935, 1994.

9. Feinbock C, Luger A, Klingler A, et al: Prospective multicentre trial comparing the efficacy of, and compliance with, glimepiride or acarbose treatment in patients with type 2 diabetes not controlled with diet alone. Diabetes Nutr Metab 16: 214-221, 2003.

10. Calles-Escandon J and Robbins DC: Loss of early phase of insulin release in humans impairs glucose tolerance and blunts thermic effect of glucose. Diabetes 36: 1167-1172, 1987.

11. Pratley RE and Weyer C: The role of impaired early insulin secretion in the pathogenesis of type II diabetes mellitus. Diabetologia 44: 929-945, 2001.

12. Del Prato S: Loss of early insulin secretion leads to postprandial hyperglycaemia. Diabetologia 46: M2-M8, 2003.

13. Owens DR, Cozma LS and Luzio SD: Early-phase prandial insulin secretion: its role in the pathogenesis of type 2 diabetes mellitus and its modulation by repaglinide. Diabet Nutr Met 15 (Suppl 6): 19-27, 2002.

14. Bruttomesso D, Pianta A, Mari A, et al: Restoration of early rise in plasma insulin levels improves the glucose tolerance of type 2 diabetic patients. Diabetes 48: 99-105, 1999.

15. Dimitriadis G, Boutati E, Lambadiari V, et al: Restoration of early insulin secretion after a meal in type 2 diabetes: effects on lipid and glucose metabolism. Eur J Clin Invest 34: 490-497, 2004.

16. Pratley RE, Foley JE and Dunning BE: Rapid acting insulinotropic agents: restoration of early insulin secretion as a physiologic approach to improve glucose control. Curr Pharm Des 7: 1375-1397, 2001 .

17. Hanefeld M, Bouter KP, Dickinson S and Guitard C: Rapid and short-acting mealtime insulin secretion with nateglinide controls both prandial and mean glycemia. Diabetes Care 23: 202-207, 2000.

18. Saloranta C, Guitard C,Pecher E, et al: Nateglinide improves early insulin secretion and controls postprandial glucose excursions in a prediabetic population. Diabetes Care 25: 2141-2146, 2002.

19. Hollander PA, Schwartz SL, Gatlin MR, et al: Importance of early insulin secretion comparison of nateglinide and glyburide in previously diet-treated patients with type 2 diabetes. Diabetes Care 24: 983-988, 2001

20. Saloranta C, Hershon K, Ball M, et al: Efficacy and safety of nateglinide in type 2 diabetic patients with modest fasting hyperglycemia. J Clin Endocrinol Metab 87: 4171-4176, 2002.

21. Gao HW, Xie C, Wang HN, et al: Beneficial metabolic effects of nateglinide versus acarbose in patients with newly-diagnosed type 2 diabetes. Acta Pharmacol Sin 28: 534-539 2007. 